\title{
Effects of concentrated long-chain omega-3 polyunsaturated fatty acid supplementation before radical prostatectomy on prostate cancer proliferation, inflammation, and quality of life: study protocol for a phase Ilb, randomized, double-blind, placebo- controlled trial
}

Marie-Hélène Guertin ${ }^{1}$, Karine Robitaille ${ }^{1}$, Jean-François Pelletier ${ }^{1}$, Thierry Duchesne², Pierre Julien³, Josée Savard', Isabelle Bairati ${ }^{1}$ and Vincent Fradet ${ }^{1 *}$

\begin{abstract}
Background: Prostate cancer is the most commonly diagnosed cancer in north-American men. Few dietary or lifestyle interventions have been tested to prevent prostate cancer progression. Omega-3 fatty acid supplementation represents a promising intervention for prostate cancer patients. The aim of the study is to evaluate the effects of longchain omega-3 polyunsaturated fatty acids (LCn3), more precisely eicosapentaenoic acid monoacylglyceride (MAG-EPA) supplementation, on prostate cancer proliferation, inflammation mediators and quality of life among men who will undergo radical prostatectomy.
\end{abstract}

Methods/design: We propose a phase Ilb, randomized, double-blind placebo-controlled trial of MAG-EPA supplementation for 130 men who will undergo radical prostatectomy as treatment for a prostate cancer of Gleason score $\geq 7$ in an academic cancer center in Quebec City. Participants will be randomized to 6 capsules of $625 \mathrm{mg}$ of fish oil (MAG-EPA) per capsule containing 500 mg of EPA daily or to identically looking capsules of high oleic acid sunflower oil (HOSO) as placebo. The intervention begins 4 to 10 weeks prior to radical prostatectomy (baseline) and continues for one year after surgery. The primary endpoint is the proliferative index (Ki-67) measured in prostate cancer cells at radical prostatectomy. A secondary endpoint includes prostate tissue levels of inflammatory mediators (cytokines and proteins) at time of radical prostatectomy. Changes in blood levels of inflammatory mediators, relative to baseline levels, at time of radical prostatectomy and 12 months after radical prostatectomy will also be evaluated. Secondary endpoints also include important aspects of psychosocial functioning and quality of life such as depression, anxiety, sleep disturbances, fatigue, cognitive complaints and prostate cancer-specific quality of life domains. The changes in these outcomes, relative to baseline levels, will be evaluated at 3, 6, 9 and 12 months after radical prostatectomy.

(Continued on next page)

\footnotetext{
* Correspondence: vincent.fradet@fmed.ulaval.ca

'Oncology Unit, Centre de recherche du CHU de Québec - Université Laval -

L'Hôtel-Dieu de Québec, 6 rue McMahon, Québec, QC, Canada

Full list of author information is available at the end of the article
} 
(Continued from previous page)

Discussion: The results from this trial will provide crucial information to clarify the role of omega-3 supplementation on prostate cancer proliferation, inflammation and quality of life.

Trial registration: ClinicalTrials.gov Identifier: NCT02333435. Registered on December 17, 2014. Last updated September 6, 2016.

Keywords: Prostate cancer, Omega-3, Proliferation, Inflammation, Quality of life

\section{Background}

Prostate cancer $(\mathrm{PCa})$ is a significant health problem worldwide. In Canada, 1 out of 8 men is expected to develop PCa in their lifetime and 1 in 27 will die from it [1]. Men diagnosed with intermediate risk PCa usually undergo radical prostatectomy (RP) or radiation therapy and have uncertain prognoses [1] and many side effects $[2,3]$. For these patients, dietary and lifestyle interventions are considered promising strategies to improve health and quality of life [4].

\section{Omega-3 fatty acids}

Long-chain omega-3 polyunsaturated fatty acids (LCn3), eicosapentaenoic acid (EPA) and docosahexanenoic acid (DHA), mainly found in seafood and fatty fish, might help lower PCa incidence and/or delay its progression [4-8]. However, some reviews reported no association [9-12] or mixed associations depending on LCn3 subtypes [13, 14]. It is important to note that observational studies are often limited by multiple sources of bias and the difficulty of estimating LCn3 intakes. LCn3 levels measured in red blood cells reflect the diet over the last 3 months [15]. However, studies that evaluated dietary LCn3 from biomarkers, assessed LCn3 in the plasma [9-11, 16-18], a measure that reflects the diet of the past few days [15]. Interestingly, we have recently measured LCn3 in the targeted prostate tissue during active surveillance of patients with a low-grade PCa. We observed a significant protective association between higher levels of EPA and a lower risk of progression to high-grade $\mathrm{PCa}$ [19].

\section{Proliferation}

Nuclear Ki-67 is a protein expressed in all proliferative phases of the cell cycle [20-23].The proliferation rate of normal prostatic epithelial cells being very low, Ki-67 is mainly expressed in PCa cells and this proliferative index is considered an important prognostic factor for PCa patients [24-27]. A Phase II randomized controlled trial (RCT) comparing a low fat diet enriched with fish oil to a western diet, in 55 men, showed that prostatic Ki-67 expression was significantly reduced in the low-fat diet/ fish oil group [28]. Flaxseed supplementation before RP was also associated with downregulation of Ki-67 in another RCT [29]. However, in these studies, proliferation was not the primary endpoint.

\section{Inflammation}

Inflammation is a highly ordered, controlled and short-lived response to infection or injury. Tumors are often viewed as "wounds that do not heal" and can prevent the proper regulation of the resolution phase of inflammation, thus taking advantage of the inflammatory process for their own benefits. The microenvironment surrounding tumors can produce and secrete several cytokines and growth factors that promote proliferation and minimize apoptosis, thus driving carcinogenesis [30, 31]. Over-expression of several inflammatory mediators in prostate tissue (e.g. IL-1 [32, 33], IL-6 [32-34], TGF- $\beta$ [35], TNF- $\alpha$ [32]) and blood (e.g. IL-6 [36], IL-7 [37] and IL-15 [37]) has been observed in $\mathrm{PCa}$ patients or $\mathrm{PCa}$ patients with progression.

LCn3, particularly EPA, have beneficial effects on systemic inflammation via modulation of the immune system, increase phagocytic activity, disruption of TLR signaling cascade and production of anti-inflammatory eicosanoids [38]. These effects are mediated by their incorporation into the plasmatic membrane. Previous studies have assessed the changes of only a limited range of systemic inflammatory mediators after omega- 3 interventions, including IL-6 [39-43], IL-1 $\beta$ [40, 41, 43-46] and TNF- $\alpha$ [42-44]. However, the effects of nutritional interventions on prostate tissue inflammation has yet to be examined using a RCT design.

\section{Quality of life and psychosocial functioning}

$\mathrm{PCa}$ and its treatment are associated with significant psychological distress. Large-scale epidemiological studies on psychological disorders in the context of $\mathrm{PCa}$ are sparse. Nonetheless, in a study conducted by our team in 861 patients treated for $\mathrm{PCa}$, we found that $17.0 \%$ exhibited clinical levels of depression, while $23.7 \%$ of the patients had clinical levels of anxiety [47]. Moreover, we observed sexual difficulties, sleep impairments and fatigue in $70.5 \%, 31.9 \%$, and $18.5 \%$ of the patients, respectively.

Epidemiological studies have shown associations between a greater annual fish intake and lower depression rates [48-50]. A RCT conducted in medical students (with no psychiatric disorder), comparing a 12-weeks LCn3 supplementation to a placebo, showed a $20 \%$ reduction of anxiety symptoms [51]. A recent study found 
no significant effect of omega-3 supplementation on sleep quality [52], while a study of 633 breast cancer survivors showed that a higher intake of omega- 6 relative to omega-3 was associated with a higher risk of fatigue [53]. These questions remain to be investigated in PCa patients using a RCT.

\section{Rationale}

Epidemiological studies point to a possible role of environmental factors, especially diet, in $\mathrm{PCa}$ incidence and progression. Evidence also suggests that an LCn3-rich diet may be beneficial to cancer patients through the modulation of cancer cell proliferation, inflammation, psychosocial functioning and quality of life. However, well conducted RCT assessing the effects of LCn3 on all these outcomes critical to PCa, are lacking. Therefore, a randomized, double-blind, placebo-controlled trial was initiated to examine the specific roles of $\mathrm{LCn} 3$ sub-type EPA on the biology and treatment consequences of PCa.

\section{Study objectives}

We hypothesize that supplementation with EPA monoacylglyceride (MAG-EPA), beginning 6 weeks (range 4 to 10 weeks) before RP and for a year after $\mathrm{RP}$, will have measurable effects on selected PCa outcomes. The specific objectives are as follows:

\section{Primary objective}

To determine the effect of daily MAG-EPA supplementation compared to placebo, on the proliferative index (nuclear Ki-67 expression) of prostate cancer cells from the RP specimen.

\section{Secondary objectives}

To determine the effect of the intervention, compared to placebo, on the targeted tissue expression of inflammatory mediators measured in the prostate tissue from the RP specimen.

To determine the effects of the intervention, compared to placebo, on blood levels, relative to baseline levels (before beginning of the intervention), of inflammatory mediators (cytokines and proteins), at the time of RP and one year after RP.

To assess the effect of the intervention, compared to placebo, on psychosocial functioning and quality of life outcomes, relative to baseline levels, at the time of RP and during the year following RP.

\section{Methods/design}

\section{Trial design and setting}

A phase IIb, randomized, double-blind, placebo-controlled trial will be conducted at the Centre Hospitalier Universitaire (CHU) de Québec - Université Laval, a supra-regional center with high surgical volume for urological cancer, particularly for prostate cancer ( $>350 \mathrm{RP}$ per year). The parallel study design is presented in Fig. 1.

\section{Recruitment}

The study is advertised in the clinic with posters and the urologists will be reminded regularly of the study. At time of diagnosis, the urologist will discuss the different treatment options with the patient. When RP is chosen as part of the treatment, the patient will be informed of the study. Then, the research nurse will present the study information and the consent form. The patient will have the needed time to decide whether he wants to participate or not to the study.

\section{Patient population and eligibility criteria Inclusion criteria}

Patients must be 18 or older, give informed consent and have chosen RP for treatment of a PCa with a Gleason score $\geq 7$.

\section{Exclusion criteria}

Patients are not eligible if they are intolerant or allergic to fish or sunflower seeds or if they have a diagnosis of bipolar disorder.

\section{Washout period}

Patients already taking omega-3 supplements can participate after a washout period of at least 8 weeks before randomization. Other dietary supplements must be stopped before randomization.

\section{Randomization/concealment/blinding}

Patients will be randomized to the intervention or placebo group 6 weeks (acceptable range 4 to 10 weeks) prior to RP. Randomization will take place at the preoperative appointment or an appointment taken specifically for the study. The randomization process will consist of a computer-generated random listing of the treatment arm using a 1:1 allocation. The randomization will be generated by the Clinical research oncology pharmacy, using permuted random blocks of 2 to 8 . Patient allocation information will be kept in a binder in a locked room of the pharmacy for the entire study period. Patients, as well as all study personnel, including outcome assessors, and medical doctors will be blinded to treatment allocation and block sizes.

\section{Intervention}

Participants assigned to the intervention will receive, for each intervention day, 6 capsules of $625 \mathrm{mg}$ of fish oil (MAG-EPA) per capsule. The supplementation is highly concentrated in EPA giving a total dosage of $3 \mathrm{~g}$ of EPA daily. The novel fish oil formulation is based on monoglycerides containing $89 \%$ of LCn3, with $80 \%$ EPA. It 


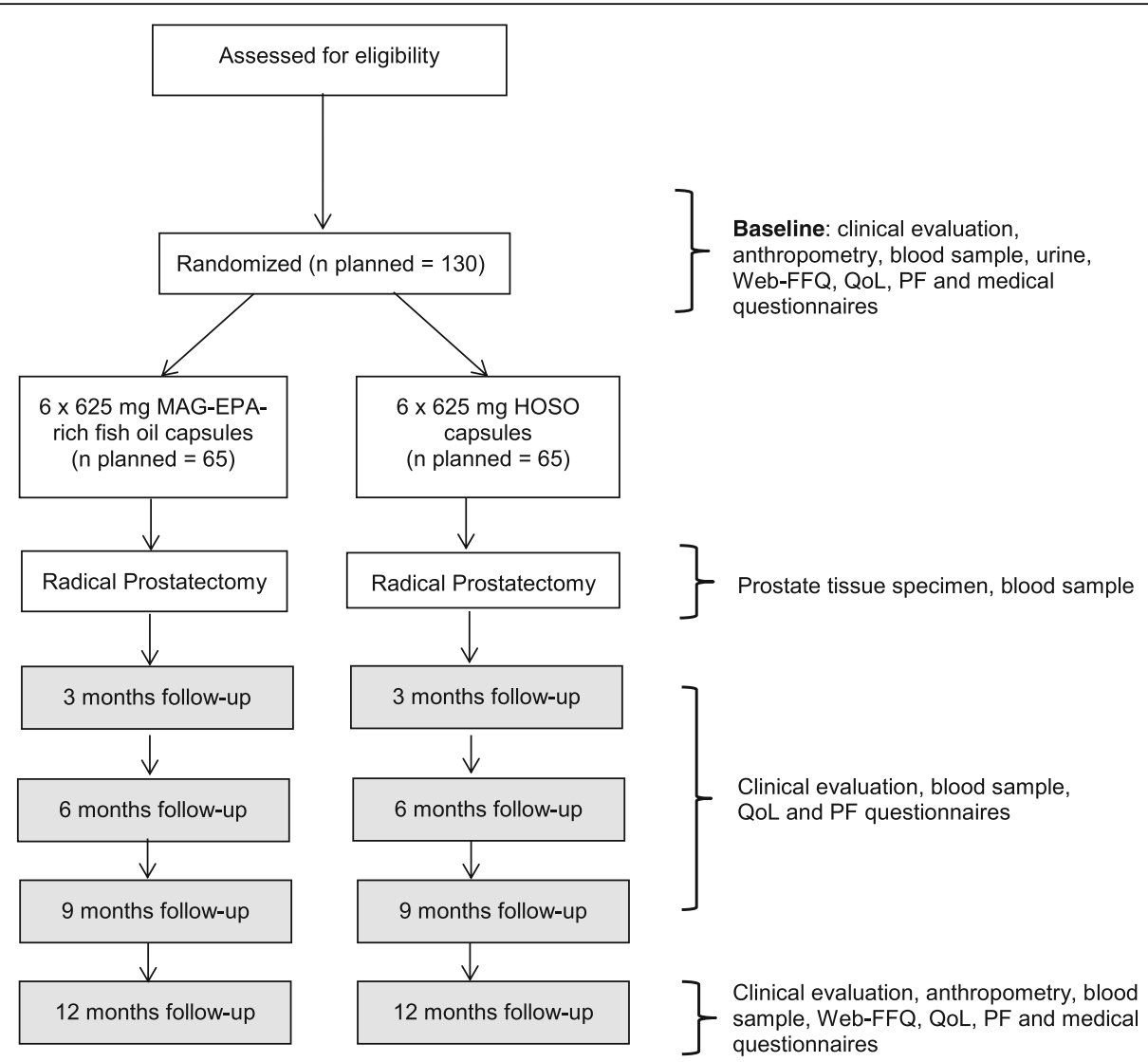

Fig. 1 Randomized Controlled Trial flow chart. 130 men diagnosed with intermediate-risk PCa treated by RP are being randomized either to LCn3 supplementation (MAG-EPA) or placebo. Intervention starts 6 weeks (acceptable range 4-10 weeks) before radical prostatectomy (RP) and ends 12 months after RP. PCa: Prostate cancer; Web-FFQ: online Food frequency questionnaire; QoL: Quality of life; PF: Psychosocial functioning; LCn3: Long chain omega-3 fatty acids; RP: Radical prostatectomy; MAG-EPA: Monoglycerides of eicosapentaenoic acid; HOSO: High oleic acid sunflower oil

presents a unique ratio of EPA/DHA of more than 10 . Most of the available products have a ratio of less than 2. It also contains approximately $3 \%$ omega- $6,3 \%$ monounsaturated and 3\% saturated fatty acids and have a less pronounced "fishy" taste compared to usual preparations.

Participants assigned to the placebo group will receive, for each intervention day, 6 capsules of identical appearance containing high oleic acid sunflower oil (HOSO). These capsules contain $82 \%$ of omega- 9 and are poor in omega- 3 or omega- 6 . This is a biologically neutral oil and has thus been used as a placebo in at least $3 \mathrm{LCn} 3$ RCTs $[43,54,55]$.

Capsules, for intervention and placebo, are prepared by SCF Pharma, Ste-Luce (Qc), and will be odorless and of identical appearance for both groups. Health Canada approved the RCT protocol and the products used for the intervention and placebo arms.

For both groups, the intervention will start 6 weeks (range 4 to 10 weeks) prior to RP and will be pursued for one year after RP. At randomization, patients receive, by the pharmacy personnel, the amount needed until their first follow-up visit, three months after surgery. They then receive, at every follow-up visit, the amount needed for the next three months until the end of follow-up.

The follow-up and care received by the patients will be the same for both groups.

\section{Concomitant medication}

Health Canada does not have any contraindication for the proposed daily dose [56]. Thus, there will be no contraindication concerning other medications taken by the patients and they will be asked to follow their usual regimen. Medications' usage will be carefully documented at the initial and subsequent visits.

\section{Data collection and follow-up}

The assessment schedule of the study is presented in Table 1. Patients will be assessed at randomization, surgery and every 3 months until one year after RP. 
Table 1 Data collection schedule

\begin{tabular}{|c|c|c|c|c|c|c|c|}
\hline & Initial visit & Randomization & $\mathrm{RP}$ & Post-RP fo & ow-up & & \\
\hline & Eligibility & $\begin{array}{l}\text { Intervention } \\
\text { (baseline - V0) }\end{array}$ & $\mathrm{V} 1$ & V2 $3 \mathrm{mo}$ & V3 $6 \mathrm{mo}$ & V4 $9 \mathrm{mo}$ & V5 $12 \mathrm{mo}$ \\
\hline Consent & & $x$ & & & & & \\
\hline Medical history and health behaviors & & $x$ & & & & & \\
\hline Concomitant medication/symptom evaluation & & $x$ & & $x$ & $x$ & $x$ & $x$ \\
\hline Consultation with uro-oncologist & $x$ & & & $x^{*}$ & $X^{*}$ & $x^{*}$ & $x^{*}$ \\
\hline Blood sample including total PSA and lipid profile & & $x$ & $x$ & $x$ & $x$ & $x$ & $x$ \\
\hline Digital rectal exam and urine sample & & $x$ & & & & & \\
\hline Radical prostatectomy (RP) & & & $x$ & & & & \\
\hline Food frequency questionnaire - FFQ online & & $x$ & & & & & $x$ \\
\hline Prostate specific quality of life (IPSS and EPIC-26) & & $x$ & & $x$ & $x$ & $x$ & $x$ \\
\hline Hospital Anxiety and Depression Scale (HADS) & & $x$ & & $x$ & $x$ & $x$ & $x$ \\
\hline $\begin{array}{l}\text { Quality of life (sleep quality, fatigue, cognitive functioning) } \\
\text { (ISI, ISF, FACT-COG V3) }\end{array}$ & & $x$ & & $x$ & $x$ & $x$ & $x$ \\
\hline General health and wellbeing (SF-36) & & $x$ & & $x$ & $x$ & $x$ & $x$ \\
\hline Fear of recurrence (FCRI) & & $x$ & & $x$ & $x$ & $x$ & $x$ \\
\hline Self-reported sleep diary & & $x$ & $x$ & $x$ & $x$ & $x$ & $x$ \\
\hline Therapeutic expectation questionnaire & & & & $x$ & & & $x$ \\
\hline Anthropometric measurements" & & $x$ & & & & & $x$ \\
\hline Vital signs ${ }^{£}$ & & $x$ & & $x$ & $x$ & $x$ & $x$ \\
\hline
\end{tabular}

RP: Radical Prostatectomy; V: visit; mo: months; PSA: Prostate Specific Antigen; IPSS: International Prostate Symptoms Score; EPIC: Expanded Prostate Index Composite; HADS: Hospital Anxiety and Depression Scale; ISI: Insomnia Severity Index; FSI: Fatigue Symptoms Inventory; Fatigue Symptoms Inventory and Functional Assessment of Cancer Therapy - Cognitive Function (FACT-COGv3); SF-36: Short-Form Health Survey; FCRI: Fear of Recurrence Inventory

*The consultation with the urologist might be on a different day but at the same period

"Height, weight, hip and waist circumference, body fat measured by skinfold caliper

${ }^{\mathrm{f}}$ Temperature, blood pressure and pulse

\section{Prostate tissue harvesting and biological specimens}

At the time of RP, immediately after surgical removal, the prostate will be kept cold $\left(4{ }^{\circ} \mathrm{C}\right)$ and transferred to the pathology unit for evaluation. A complete crosssection of the prostate will be harvested by the pathologist. The cross-section will be divided in four specific quadrants before Optimal cutting temperature (OCT) compound freezing procedure. A small portion of each quadrant (approximately $10 \mathrm{mg}$ ) will be harvested from the cross-section and snap frozen separately. All prostate tissues are stored at $-80{ }^{\circ} \mathrm{C}$.

$50 \mathrm{ml}$ of blood will be collected at indicated timepoints (Table 1). Plasma, buffy coat, red blood cells, and serum will be processed from $35 \mathrm{~mL}$ of blood collected. All samples will be stored at $-80{ }^{\circ} \mathrm{C}$. Urine post-digital rectal examination will be collected at initial visit and stored in several buffers at $-80{ }^{\circ} \mathrm{C}$.

Procedures used in this research followed the Standard operating procedures (SOPs) of the Canadian Tissue Repository Network (CTRNet). All biological specimens will be stored at the Centre de recherche du CHU de Québec - Université Laval - L'Hôtel-Dieu de Québec hospital and all patients will have provided consent for long-term storage of their tissue.
Detailed information about samples, volume collected and aliquot is provided in the (see Additional file 1: Table S1).

\section{Outcomes}

Primary outcome

The primary outcome is cancer proliferation. After immunostaining for Ki-67, slides will be scanned using an automated slide scanner and the proportion of cancer cells with positive nuclear staining will be automatically quantified using algorithms we previously developed with the Calopix (Tribvn) software [27]. Initial results in PCa tissue from RP specimens at our institution showed a linear correlation between automated quantification and visual evaluation [27]. This PCa proliferative index was also an independent predictor of prostate cancer specific mortality, making this outcome clinically relevant.

\section{Secondary outcomes}

Many inflammation mediators (Il-2, IL-8, IL-10, IL-12, IFN- $\gamma$, to name a few) will be measured in the prostatic tissue and in circulation using a Bio-plex Precision Pro kit (Bio-Rad, Toronto, On). The limit of sensitivity (LoS) 
ranges from $0.2-2.7 \mathrm{pg} / \mathrm{mL}$, allowing for greater probability of detecting the cytokines of interest, if expressed, than usual multiplexing kits (LoS of $2-5 \mathrm{pg} / \mathrm{mL}$ ).

Prostate specific aspects of quality of life is assessed using the International Prostate Symptoms Score (IPSS) and the Expanded Prostate Index Composite score (EPIC-26) for which the French-Canadian version has been validated $[57,58]$. The IPSS contains 7 questions concerning lower urinary tract symptoms for which severity is scored on a $0-5$ points scale. It also contains one quality of life item scored on a scale ranging from 0 to 6 . EPIC-26 contains 5 quality of life $\mathrm{PCa}$-specific domains: urinary incontinence, urinary irritation/obstruction, bowel, vitality/hormonal, and sexual function. Each domain is scored on a scale from 0 to 100 .

Anxiety and depression are assessed using the FrenchCanadian version of the Hospital Anxiety and Depression Scale (HADS) for which we observed excellent internal consistency and test-retest reliability [59]. The questionnaire includes 14 items scored on a scale range from 0 to 3 , with 7 items assessing anxiety and 7 items assessing depression.

Fatigue related quality of life is assessed using French versions of the Insomnia Severity Index (ISI) [60] and the Fatigue Symptoms Inventory (FSI) [61]. The ISI enquires about sleep quality over the past two weeks and contains 7 items scored on a 0 to 4 scale. Good psychometric properties have been reported [62]. The FSI questionnaire assesses, for the past week, fatigue intensity (4 items), fatigue duration (2 items) and how fatigue interfered with quality of life (7 items). The questionnaire exhibited high internal consistency and construct validity [61].

Cognitive function was also evaluated with the Functional Assessment of Cancer Therapy - Cognitive Function (FACT-COG v3) [63]. The questionnaire contains 37 items covering 4 subscales. The subscale includes Perceived cognitive impairments, Comments from others, Perceived cognitive abilities and Impact on quality of life. Each item is scored on a 5-points Likert scale ranging with a range of 0 to 4 . This tool also showed high consistency and validity [63].

To assess cancer-specific anxiety, fear of recurrence is evaluated using the 9 items of the severity subscale of the Fear of Cancer Recurrence Inventory (FCRI) which was validated in french [64]. The items are scored from 0 to 4 and assess the presence, frequency and intensity of thoughts associated with cancer recurrence.

Finally, general wellbeing is evaluated using the French version of the Short-Form Health Survey, SF-36, (SF-36v2 Standard, Canada (French) Version 2.0) [65].

\section{Confounding variables}

Potential confounders will be measured at baseline, before the beginning of the intervention. Age, anthropometry, medical history, cancer stage, clinical and pathological grade (Gleason), alcohol and tobacco use will be assessed.

Physical activity for a typical 7-day period will also be assessed at baseline by the Godin Leisure-Time Exercise Questionnaire [66]. The questionnaire contains 3 items assessing the number of times engaging in mild, moderate and strenuous exercises for at least $15 \mathrm{~min}$. One item evaluates the number of times one engages in any activity long enough to work up a sweat.

Dietary intake in the past month will be measured using a web-based self-administered food frequency questionnaire (web-FFQ), which contains 136 questions and 40 sub-questions covering 8 food categories including the four groups of the Canadian Food Guide. The questionnaire has been specifically developed for the population of Quebec and validated in healthy men [67]. We also specifically validated the assessment of omega- 3 intake in a population of PCa patients [68]. Dietary intake will be assessed at baseline and 12 months post-RP.

\section{Adherence and therapeutic expectations}

The pharmacy personnel will monitor adherence by counting remaining pills. The success of blinding will be evaluated using a therapeutic expectations questionnaire administered at 3 and 12 months post-RP.

\section{Sample size calculation}

Sample size analysis was done using a two-sample t-test for a log-normal geometric mean ratio with a two-sided significance level of 0.05 , assuming equal variances. Based on published data $[28,29]$, we assume a coefficient of variation of 0.4 . In the study conducted by Aronson et al. [28], a statistically significant reduction of $32 \%$ in the proportion of cells expressing Ki-67 was observed in a group receiving a low-fat diet supplemented with fish oil compared to a control group assigned to a Western diet. We determined that, for the primary outcome, a total of 126 patients (63/group) will provide $90 \%$ power $[69,70]$ to detect a mean ratio of the proportions of cancer cells expressing Ki-67 of $\leq 0.8$, i.e. a $20 \%$ difference across groups. We estimate $\leq 3 \%$ drop out before RP (occurring in $\leq 1 \%$ at our institution). Based on these estimation, the sample size needed for the trial will be 130 patients (65/group).

Twelve months after RP, we expect a 5 to $10 \%$ loss to follow-up, which will affect only secondary outcomes. In absence of published data on the effects of omega-3 supplementation on inflammation mediators, we used Cohen's $d$ to calculate effect sizes [71]. A total sample size of 116 will provide $80 \%$ statistical power to detect a moderate or large effect size $(\geq 0.5)$. This sample size will also be sufficient to detect expected between-groups differences on quality of life dimensions. Indeed, LCn3 supplementation reduced anxiety by $20 \%(\mathrm{~d}=0.51)$ in 
one study [51] and it yielded a moderate effect size $(\mathrm{d}=0.61)$ for treating depressive disorders (vs. placebo) in another [72]. For these effect sizes in our final sample, we evaluate a power of $80 \%$ to detect an effect for anxiety and a power of $92 \%$ to detect an effect for depressive symptoms.

\section{Statistical analyses \\ Primary outcome}

The average proportion of cells expressing Ki-67 in each group will be compared, using the t-test, after transformation of the data if the population is not normally distributed. The Wilcoxon rank sum test will be used if observations are not normally distributed after transformation procedures. Multivariable linear regression will be carried out in the event of unbalanced distributions of important baseline characteristics such as cancer grade and stage, age, baseline inflammatory levels, diet or health behaviors. The intervention leading to the primary outcome is of a relatively short duration (4-10 weeks) and since almost all recruited patients are expected to undergo prostatectomy, few missing data are expected. The analyses will include all randomized patients for whom a ki-67 is measurable.

A per protocol analysis will also be carried out as exploratory analyses. These analyses will include participants with a measurable ki-67 and who will have taken at least $80 \%$ of intervention or placebo doses.

For all analyses, bilateral $p$-values of less than 0.05 will be considered statistically significant.

\section{Secondary outcomes}

For the secondary outcome pertaining to prostatic levels of inflammation mediators, the t-tests (or Wilcoxon rank-sum test) will be used to compare levels across groups.

Blood levels of inflammation biomarkers as well as outcomes concerning quality of life are measured at multiple time points. For these analyses, linear mixed models, to take into account for multiple measurements, will be performed.

Confounding factors will also be taken into account in the analyses in the case of unbalanced distribution between groups.

\section{Subgroup analyses}

It is possible that some patients will already have high dietary intake of omega-3. Subgroups analyses will be carried out to explore how baseline levels of omega-3 in the diet can modify the effect of the intervention. The effect of the intervention will be tested for low or high levels of total omega-3 (and LCn3) using intake with an interaction term in the multivariable linear regression.
The levels measured in red blood cells, a good proxy to the diet, will also be evaluated. The cutoff will be the recommended dietary intake of $1.6 \mathrm{~g}$ daily [73] for total omega- 3 and $250 \mathrm{mg}$ for EPA (the equivalent of approximately $8 \mathrm{oz}$ per week of a variety of seafood [74]). For red blood cells levels, the value corresponding to the recommended dietary intake (modeled in this cohort) will be used as a cutoff.

\section{Ethical considerations}

The study protocol has been approved by the ethics committee of CHU de Québec - Université Laval (2012-1012). Written informed consent is obtained from all randomized patients for the study as well as for biobanking of their biological specimens (blood, urine, tissue).

\section{Adverse events safety monitoring}

Many studies have already shown LCn3 safety. Therefore, no major harmful effects are expected. However, adverse events will be monitored at each visit post-prostatectomy using the NIH criteria for adverse events (v4.03, June 2010). An independent safety monitoring board includes a statistician Dr. Benoit Masse (PhD), Head of Applied Clinical Research Unit of CHU Sainte-Justine Research Center, Montréal, Quebec and a prostate cancer and clinical research trial expert, Dr. Fred Saad (MD), Centre Hospitalier de l'Université de Montréal Research Center, Montreal, Quebec.

\section{Data collection, managing and monitoring}

All data is collected at the research center by research professionals. Questionnaires are scanned and validated by a research professional. The data is stored on the network of the Centre de recherche du CHU de Québec - Université Laval following their Standard Operative Procedures to ensure confidentiality. Only the PI and the research professionals implied in the current study will have access to the data and protected by a password. Questionnaires will be stored in a locked filing cabinet which is in a locked room. Data collection follow-up monitoring will be realized on a regular basis by the research team.

\section{Discussion}

This trial is the first to examine the effects of preoperative LCn3 supplementation with $\mathrm{Ki}-67$ as a primary outcome. Moreover, it is, to our knowledge, the first to examine the effects of $\mathrm{LCn} 3$ on inflammation and quality of life among PCa patients. Well conducted RCTs assessing the effect of LCn3 on PCa outcomes are important as conflicting results from observational studies do not allow conclusions to be drawn so far. In fact, the need for prospective RCTs to identify specific nutrients for $\mathrm{PCa}$ patients has 
recently been highlighted [4]. This trial will contribute to the improvement of available evidence for clinicians and $\mathrm{PCa}$ patients who want reliable information on lifestyle strategies to improve survivorship and quality of life [75]. PCa patients would benefit from interventions harbouring few or no side effects. As such, lifestyle and nutritional interventions are appealing as they are innocuous and have the potential to improve various clinically relevant outcomes. High quality trials are important to allow these interventions to be offered to patients in combination with other established treatments.

\section{Status of the trial}

The first patient was enrolled on February 12, 2015. The enrollment and randomization of the planned $130 \mathrm{pa}-$ tients was completed on June 9, 2017. The data collection is expected to end in August 2018.

\section{Additional file}

Additional file 1: Table S1. Trial biospecimen collection. The table contains detailed information on samples with volumes collected and aliquot (DOCX $15 \mathrm{~kb})$

\section{Abbreviations}

EPIC-26: Expanded Prostate Index Composite score; FACT-COG: Functional Assessment of Cancer Therapy - Cognitive Function; FCRI: Fear of Cancer Recurrence Inventory; FFQ: Food frequency questionnaire; FSI: Fatigue symptoms inventory; HADS: Hospital Anxiety and Depression Scale; HOSO: high oleic acid sunflower oil; IPSS: International Prostate Symptoms Score; LCn3: long-chain omega-3 polyunsaturated fatty acids; LOS: Limit of sensitivity; MAG-EPA: eicosapentaenoic acid monoacylglyceride; OCT: Optimal cutting temperature; PCa: Prostate cancer; RCT: Randomized controlled trial; RP: Radical prostatectomy; SOP: Standard operating procedures

\section{Acknowledgements}

The authors thank the research nurses and research professionals from the CHU de Québec - Université Laval involved in the study, especially Hélène Hovington for the management of data collection and database. Dr. Benoitt Mâsse and Dr. Fred Saad are thanked for their role in the Data and Safety Monitoring Board. A career Junior 2 award from the Fonds de recherche du Québec - Santé supported Dr. Vincent Fradet.

\section{Funding}

This study was funded by the Lotte and John Hecht Memorial Foundation Innovation Grant of the Canadian Cancer Society Research Institute (grant \#2012-702,569). The funding body, as well as SCF Pharma and Solutex, have no role in the design, collection, analysis, interpretation and publication of the data.

\section{Availability of data and materials}

Not applicable. No data analyses are presented.

\section{Authors' contributions}

$V F$ is the grant principal investigator and IB, JS, TD and PJ are COinvestigators. They all contributed to the development of the study protocol. VF and KR are responsible for study management. KR and MHG contributed to the literature review and manuscript writing. JFP is responsible for biobanking and overseeing data collection. All authors contributed to the revision and approval of the manuscript.

\section{Ethics approval and consent to participate}

The study protocol has been approved by the ethics committee of $\mathrm{CHU}$ de Québec - Université Laval (2012-1012). Written informed consent was obtained from all recruitment patients for study participation as well as for biobanking of their biological specimen (blood, urine, tissue).

\section{Consent for publication}

Not applicable.

\section{Competing interests}

The authors declare that they have no competing interests. SCF Pharma and Solutex (Spain) provide capsules (intervention and placebo) for the study,

free of charge. They, not have any competing interests regarding our study.

\section{Publisher's Note}

Springer Nature remains neutral with regard to jurisdictional claims in published maps and institutional affiliations.

\section{Author details}

'Oncology Unit, Centre de recherche du CHU de Québec - Université Laval L'Hôtel-Dieu de Québec, 6 rue McMahon, Québec, QC, Canada.

${ }^{2}$ Mathematics and Statistics Department, Université Laval, 1045 avenue de la médecine, Bureau, Québec, QC 1056, Canada. ${ }^{3}$ Endocrinology and Nephrology Unit, Centre de recherche du CHU de Québec - Université Laval - CHUL, 2705, boulevard Laurier, Québec, QC, Canada.

Received: 28 June 2017 Accepted: 22 December 2017

Published online: 10 January 2018

\section{References}

1. Prostate Cancer Statistics. Canadian Cancer Society, 2017. (Accessed March 28 2017, at http://www.cancer.ca/en/cancer-information/cancer-type/ prostate/statistics/?region=on.)

2. Donovan IL, Hamdy FC, Lane JA, et al. Patient-reported outcomes after monitoring, surgery, or radiotherapy for prostate cancer. N Engl J Med. 2016:375:1425-37.

3. Fossa SD, Bengtsson T, Borre M, Ahlgren G, Rannikko A, Dahl AA. Reduction of quality of life in prostate cancer patients: experience among 6200 men in the Nordic countries. Scand J Urol. 2016;50:330-7.

4. Lin PH, Aronson W, Freedland SJ. Nutrition, dietary interventions and prostate cancer: the latest evidence. BMC Med. 2015;13:3.

5. Reese AC, Fradet V, Witte JS. Omega-3 fatty acids, genetic variants in COX-2 and prostate cancer. J Nutrigenet Nutrigenomics. 2009;2:149-58.

6. Mauermann J, Pouliot F, Fradet V. Dietary omega-3 fatty acids, genetic variation and risk of breast and prostate cancers. World Rev Nutr Diet. 2011; 102:156-71

7. Szymanski KM, Wheeler DC, Mucci LA. Fish consumption and prostate cancer risk: a review and meta-analysis. Am J Clin Nutr. 2010;92:1223-33.

8. Masko EM, Allott EH, Freedland SJ. The relationship between nutrition and prostate cancer: is more always better? Eur Urol. 2013;63:810-20.

9. Gerber M. Omega-3 fatty acids and cancers: a systematic update review of epidemiological studies. Br J Nutr. 2012;107(Suppl 2):S228-39.

10. Alexander DD, Bassett JK, Weed DL, Barrett EC, Watson H, Harris W. Metaanalysis of long-chain Omega-3 polyunsaturated fatty acids (LComega3PUFA) and prostate cancer. Nutr Cancer. 2015;67:543-54.

11. Dinwiddie MT, Terry PD, Whelan J, Patzer RE. Omega-3 fatty acid consumption and prostate cancer: a review of exposure measures and results of epidemiological studies. J Am Coll Nutr. 2016;35:452-68.

12. Aucoin M, Cooley $K$, Knee $C$, et al. Fish-derived Omega-3 fatty acids and prostate cancer: a systematic review. Integr Cancer Ther. 2016;

13. Fu YQ, Zheng JS, Yang B, Li D. Effect of individual omega-3 fatty acids on the risk of prostate cancer: a systematic review and dose-response metaanalysis of prospective cohort studies. J Epidemiol. 2015;25:261-74.

14. Chua ME, Sio MC, Sorongon MC, Dy JS. Relationship of dietary intake of omega-3 and omega- 6 fatty acids with risk of prostate cancer development: a meta-analysis of prospective studies and review of literature. Prostate Cancer. 2012;2012:826254.

15. Serra-Majem L, Nissensohn M, Overby NC, Fekete K. Dietary methods and biomarkers of omega 3 fatty acids: a systematic review. Br J Nutr 2012;107 Suppl 2:S64-S76. 
16. Crowe FL, Appleby PN, Travis RC, et al. Circulating fatty acids and prostate cancer risk: individual participant meta-analysis of prospective studies. J Nat Cancer Inst. 2014;106

17. Brasky TM, Till $C$, white $E$, et al. serum phospholipid fatty acids and prostate cancer risk: results from the prostate cancer prevention trial. Am J Epidemiol. 2011;173:1429-39.

18. Brasky TM, Darke AK, Song X, et al. Plasma phospholipid fatty acids and prostate cancer risk in the SELECT trial. J Natl Cancer Inst. 2013;105:1132-41.

19. Moreel $X$, Allaire J, Leger $C$, et al. Prostatic and dietary omega-3 fatty acids and prostate cancer progression during active surveillance. Cancer Prev Res (Phila). 2014;7:766-76.

20. Bettencourt MC, Bauer JJ, Sesterhenn IA, Mostofi FK, MCLeod DG, Moul JW $\mathrm{Ki}-67$ expression is a prognostic marker of prostate cancer recurrence after radical prostatectomy. J Urol. 1996;156:1064-8.

21. Bubendorf $L$, Sauter $G$, Moch $H$, et al. Ki67 labelling index: an independent predictor of progression in prostate cancer treated by radical prostatectomy. J Pathol. 1996:178:437-41.

22. Inoue T, Segawa T, Shiraishi T, et al. Androgen receptor, Ki67, and p53 expression in radical prostatectomy specimens predict treatment failure in Japanese population. Urology. 2005;66:332-7.

23. Nariculam J, Freeman A, Bott $\mathrm{S}$, et al. Utility of tissue microarrays for profiling prognostic biomarkers in clinically localized prostate cancer: the expression of $\mathrm{BCL}-2$, E-cadherin, $\mathrm{Ki}-67$ and p53 as predictors of biochemical failure after radical prostatectomy with nested control for clinical and pathological risk factors. Asian J Androl. 2009;11:109-18.

24. Sulik M, Guzinska-Ustymowicz K. Expression of Ki-67 and PCNA as proliferating markers in prostate cancer. Rocz Akad Med Bialymst. 2002;47:262-9.

25. Kim SH, Park WS, Park BR, et al. PSCA, cox-2, and Ki-67 are independent, predictive markers of biochemical recurrence in clinically localized prostate cancer: a retrospective study. Asian J Androl. 2016;

26. Verma R, Gupta V, Singh J, et al. Significance of p53 and ki-67 expression in prostate cancer. Urol Ann. 2015;7:488-93.

27. Desmeules $P$, Hovington $H$, Nguile-Makao M, et al. Comparison of digital image analysis and visual scoring of $\mathrm{KI}-67$ in prostate cancer prognosis after prostatectomy. Diagn Pathol. 2015;10:67

28. Aronson WJ, Kobayashi N, Barnard RJ, et al. Phase II prospective randomized trial of a low-fat diet with fish oil supplementation in men undergoing radical prostatectomy. Cancer Prev Res (Phila). 2011:4:2062-71.

29. Demark-Wahnefried W, Polascik TJ, George SL, et al. Flaxseed supplementation (not dietary fat restriction) reduces prostate cancer proliferation rates in men presurgery. Cancer Epidemiol Biomark Prev. 2008; 17:3577-87

30. De Marzo AM, DeWeese TL, Platz EA, et al. Pathological and molecular mechanisms of prostate carcinogenesis: implications for diagnosis, detection, prevention, and treatment. J Cell Biochem. 2004;91:459-77.

31. Sciarra A, Mariotti G, Salciccia S, et al. Prostate growth and inflammation. J Steroid Biochem Mol Biol. 2008;108:254-60.

32. Bouraoui Y, Ricote M, Garcia-Tunon I, et al. Pro-inflammatory cytokines and prostate-specific antigen in hyperplasia and human prostate cancer. Cancer Detect Prev. 2008:32:23-32

33. Sfanos KS, De Marzo AM. Prostate cancer and inflammation: the evidence. Histopathology. 2012;60:199-215.

34. Mechergui YB, Ben Jemaa A, Mezigh C, et al. The profile of prostate epithelial cytokines and its impact on sera prostate specific antigen levels. Inflammation. 2009:32:202-10.

35. Schroten C, Dits NF, Steyerberg EW, et al. The additional value of TGFbeta1 and IL-7 to predict the course of prostate cancer progression. Cancer Immunol Immunother. 2012;61:905-10.

36. Stark JR, Li H, Kraft P, et al. Circulating prediagnostic interleukin-6 and Creactive protein and prostate cancer incidence and mortality. Int J Cancer. 2009;124:2683-9.

37. Mengus C, Le Magnen C, Trella E, et al. Elevated levels of circulating IL-7 and IL-15 in patients with early stage prostate cancer. J Transl Med. 2011;9:162.

38. de Pablo MA, Alvarez de Cienfuegos G. Modulatory effects of dietary lipids on immune system functions. Immunol Cell Biol. 2000;78:31-9.

39. Abbate R, Gori AM, Martini F, et al. N-3 PUFA supplementation, monocyte PCA expression and interleukin-6 production. Prostaglandins Leukot Essent Fatty Acids. 1996;54:439-44.

40. Blok WL, Deslypere JP, Demacker PN, et al. Pro- and anti-inflammatory cytokines in healthy volunteers fed various doses of fish oil for 1 year. Eur J Clin Investig. 1997;27:1003-8.
41. Cooper AL, Gibbons L, Horan MA, Little RA, Rothwell NJ. Effect of dietary fish oil supplementation on fever and cytokine production in human volunteers. Clin Nutr. 1993;12:321-8.

42. Trebble T, Arden NK, Stroud MA, et al. Inhibition of tumour necrosis factoralpha and interleukin 6 production by mononuclear cells following dietary fish-oil supplementation in healthy men and response to antioxidant cosupplementation. Br J Nutr. 2003;90:405-12.

43. Pot GK, Brouwer IA, Enneman A, Rijkers GT, Kampman E, Geelen A. No effect of fish oil supplementation on serum inflammatory markers and their interrelationships: a randomized controlled trial in healthy, middle-aged individuals. Eur J Clin Nutr. 2009:63:1353-9.

44. Caughey GE, Mantzioris E, Gibson RA, Cleland LG, James MJ. The effect on human tumor necrosis factor alpha and interleukin 1 beta production of diets enriched in n-3 fatty acids from vegetable oil or fish oil. Am J Clin Nutr. 1996:63:116-22.

45. Endres S, Ghorbani R, Kelley VE, et al. The effect of dietary supplementation with n-3 polyunsaturated fatty acids on the synthesis of interleukin-1 and tumor necrosis factor by mononuclear cells. N Engl J Med. 1989;320:265-71.

46. Gallai $\bigvee$, Sarchielli $P$, Trequattrini A, et al. Cytokine secretion and eicosanoid production in the peripheral blood mononuclear cells of MS patients undergoing dietary supplementation with n-3 polyunsaturated fatty acids. J Neuroimmunol. 1995;56:143-53.

47. Hervouet S, Savard J, Simard S, et al. Psychological functioning associated with prostate cancer: cross-sectional comparison of patient treated with radiotherapy, brachytherapy, or surgery. J Pain Symptom Manag. 2005;30:474-84

48. Hoffmire CA, Block RC, Thevenet-Morrison K, van Wijngaarden E. Associations between omega-3 poly-unsaturated fatty acids from fish consumption and severity of depressive symptoms: an analysis of the 20052008 National Health and nutrition examination survey. Prostaglandins Leukot Essent Fatty Acids. 2012;86:155-60.

49. McNamara RK. Evaluation of docosahexaenoic acid deficiency as a preventable risk factor for recurrent affective disorders: current status, future directions, and dietary recommendations. Prostaglandins Leukot Essent Fatty Acids. 2009;81:223-31.

50. McNamara RK. Role of Omega-3 fatty acids in the etiology, treatment, and prevention of depression: current status and future directions. J Nutr Intermed Metab. 2016;5:96-106.

51. Kiecolt-Glaser JK, Belury MA, Andridge R, Malarkey WB, Glaser R. Omega-3 supplementation lowers inflammation and anxiety in medical students: a randomized controlled trial. Brain Behav Immun. 2011;25:1725-34.

52. Kiecolt-Glaser JK, Belury MA, Andridge R, Malarkey WB, Hwang BS, Glaser R. Omega-3 supplementation lowers inflammation in healthy middle-aged and older adults: a randomized controlled trial. Brain Behav Immun. 2012;26:988-95.

53. Alfano $\mathrm{CM}$, Imayama I, Neuhouser $\mathrm{ML}$, et al. Fatique, inflammation, and omega-3 and omega- 6 fatty acid intake among breast cancer survivors. J Clin Oncol. 2012;30:1280-7.

54. de Roos B, Geelen A, Ross K, et al. Identification of potential serum biomarkers of inflammation and lipid modulation that are altered by fish oil supplementation in healthy volunteers. Proteomics. 2008:8:1965-74.

55. Ottestad I, Vogt G, Retterstol K, et al. Oxidised fish oil does not influence established markers of oxidative stress in healthy human subjects: a randomised controlled trial. Br J Nutr. 2012;108:315-26.

56. Monograph: Fish Oil. Health Canada; 2015.

57. Szymanski KM, Wei JT, Dunn RL, Sanda MG. Development and validation of an abbreviated version of the expanded prostate cancer index composite instrument for measuring health-related quality of life among prostate cancer survivors. Urology. 2010;76:1245-50.

58. Vigneault $\mathrm{E}$, Savard J, Ivers $\mathrm{H}$, et al. Validation of a French-Canadian Version of the Expanded Prostate Cancer Index Composite Instrument. Can Urol Ass J 2017; In Press.

59. Savard J, Laberge B, Gauthier JG, Ivers H, Bergeron MG. Evaluating anxiety and depression in HIV-infected patients. J Pers Assess. 1998;71:349-67.

60. Morin $\mathrm{CM}$, Belleville $\mathrm{G}$, Belanger $\mathrm{L}$, Ivers $\mathrm{H}$. The insomnia severity index: psychometric indicators to detect insomnia cases and evaluate treatment response. Sleep. 2011:34:601-8.

61. Hann DM, Denniston MM, Baker F. Measurement of fatigue in cancer patients: further validation of the fatigue symptom inventory. Qual Life Res. 2000;9:847-54

62. Bastien $\mathrm{CH}$, Vallieres $\mathrm{A}$, Morin $\mathrm{CM}$. Validation of the insomnia severity index as an outcome measure for insomnia research. Sleep Med. 2001;2:297-307. 
63. Cella DF, Tulsky DS, Gray G, et al. The functional assessment of cancer therapy scale: development and validation of the general measure. J Clin Oncol. 1993;11:570-9.

64. Simard S, Savard J. Fear of cancer recurrence inventory: development and initial validation of a multidimensional measure of fear of cancer recurrence. Support Care Cancer. 2009:17:241-51.

65. Ware JE Jr, Sherbourne CD. The MOS 36-item short-form health survey (SF36). I. Conceptual framework and item selection. Med Care. 1992;30:473-83.

66. Godin G, Shephard R. Godin Leisure-Time Exercise Questionnaire. Med Sci Sports Exerc. 1997;29:S36-S8.

67. Labonte ME, Cyr A, Baril-Gravel L, Royer MM, Lamarche B. Validity and reproducibility of a web-based, self-administered food frequency questionnaire. Eur J Clin Nutr. 2012;66:166-73.

68. Allaire J, Moreel X, Labonte ME, et al. Validation of the omega-3 fatty acid intake measured by a web-based food frequency questionnaire against omega-3 fatty acids in red blood cells in men with prostate cancer. Eur J Clin Nutr. 2015;69:1004-8.

69. O'Brien RG, Muller KE. Unified power analysis for t-tests through multivariate hypothesis. In: Edwards LK, editor. Applied analysis of variance in the behavioral sciences. New York: Marcel Dekker; 1993. p. 297-344.

70. Self SG, Mauritsen $\mathrm{RH}$, Ohara J. Power calculations for the likelihood ratio tests in generalized linear models. Biometrics. 1992:48:31-9.

71. Kirk R. Experimental design: procedures for the behavioral sciences. Pacific Grove, Calif: Brooks/Cole; 1995

72. Lin PY, Mischoulon D, Freeman MP, et al. Are omega-3 fatty acids antidepressants or just mood-improving agents? The effect depends upon diagnosis, supplement preparation, and severity of depression. Mol Psychiatry. 2012;17:1161-3. author reply 3-7

73. Institute of Medicine, Food and Nutrition Board. Dietary reference intakes for energy, carbohydrate, fiber, fat, fatty acids, cholesterol, protein, and amino acids (macronutrients). Washington, DC: National Academy Press; 2005.

74. U.S. Department of Health and Human Services, U.S. Department of Agriculture. 2015-2020 dietary guidelines for Americans. 2015.

75. Avery KN, Donovan JL, Horwood J, et al. The importance of dietary change for men diagnosed with and at risk of prostate cancer: a multi-centre interview study with men, their partners and health professionals. BMC Fam Pract. 2014;15:81.

\section{Submit your next manuscript to BioMed Central and we will help you at every step:}

- We accept pre-submission inquiries

- Our selector tool helps you to find the most relevant journal

- We provide round the clock customer support

- Convenient online submission

- Thorough peer review

- Inclusion in PubMed and all major indexing services

- Maximum visibility for your research

Submit your manuscript at www.biomedcentral.com/submit 